\title{
Tipifarnib in the treatment of newly diagnosed acute myelogenous leukemia
}

\author{
Judith E Karp' \\ Jeffrey E Lancet ${ }^{2}$ \\ 'Division of Hematologic Malignancies, \\ Johns Hopkins Sidney Kimmel \\ Comprehensive Cancer Center, \\ Baltimore, Maryland, USA; \\ ${ }^{2} \mathrm{H}$. Lee Moffitt Comprehensive \\ Cancer Center, Tampa, Florida, USA
}

Correspondence: Judith E Karp

Director, Leukemia Program

Division of Hematologic Malignancies

Johns Hopkins Sidney Kimmel

Comprehensive Cancer Center

I650 Orleans Street, CRB I Room 289

Baltimore, Maryland 21231-1000

Tel + I 4105027726

Fax +I 4I0614 I005

Email jkarp2@jhmi.edu

\begin{abstract}
Farnesyltransferase inhibitors (FTIs) represent a new class of signal transduction inhibitors that block the processing of cellular polypeptides that have cysteine terminal residues and, by so doing, interdict multiple pathways involved in proliferation and survival of diverse malignant cell types. Tipifarnib is an orally bioavailable, nonpeptidomimetic methylquinolone FTI that has exhibited clinical activity in patients with myeloid malignancies including elderly adults with acute myelogenous leukemia (AML) who are not candidates for traditional cytotoxic chemotherapy, patients with high-risk myelodysplasia, myeloproliferative disorders, and imatinibresistant chronic myelogenous leukemia. Because of its relatively low toxicity profile, tipifarnib provides an important alternative to traditional cytotoxic approaches for elderly patients who are not likely to tolerate or even benefit from aggressive chemotherapy. In this review, we will focus on the clinical development of tipifarnib for treatment of newly diagnosed AML, both as induction therapy for elderly adults with poor-risk AML and as maintenance therapy following achievement of first complete remission following induction and consolidation therapies for poor-risk AML. As with all other malignancies, the optimal approach is likely to lie in rational combinations of tipifarnib with cytotoxic, biologic and/or immunomodulatory agents with non-cross-resistant mechanisms of action. Gene expression profiling has identified networks of differentially expressed genes and gene combinations capable of predicting response to single agent tipifarnib. The clinical and correlative laboratory trials in progress and under development will provide the critical foundations for defining the optimal roles of tipifarnib and in patients with AMl and other hematologic malignancies.
\end{abstract}

Keywords: farnesylation, farnesyltransferase inhibitor, acute myelogenous leukemia (AML), signal transduction, gene expression, tipifarnib

Cure rates for adults with acute myelogenous leukemia (AML) remain inadequate. Poor-risk features such as age $\geq 60$, secondary AML including prior myelodysplasia (MDS) and treatment-related AML (t-AML), adverse cytogenetics, and hyperleukocytosis with extramedullary disease in the absence of "favorable" cytogenetics identify patients who are not only less likely to achieve remissions with cytotoxic chemotherapy but who are predisposed to shorter disease free survival despite intensive induction and post-remission therapies (Lancet et al 2000; Grimwade et al 2001; Goldstone et al 2001; Appelbaum et al 2006; Estey 2007).

In particular, the response rates and survival for adults age 70 and older with AML remain limited. Part of this poor response relates to the inability of very elderly patients (age $\geq 75$ ) to tolerate intensive chemotherapy (Lancet et al 2000; Appelbaum et al 2006; Estey 2007). Equally important, however, is the genetic complexity and inherent resistance of these AMLs to the cytotoxic effects of traditional cytotoxic agents (Grimwade et al 2001; Goldstone et al 2001; Appelbaum et al 2006). AMLs that arise in the older age group often do so from an antecedent hematologic disorder, mainly MDS which itself has evolved from a background of 
toxin exposures (Grimwade et al 2001; Appelbaum et al 2006; Estey 2007). These MDS/AMLs exhibit a complex genetic profile, likely a result of cumulative genomic damage (Grimwade et al 2001).

Farnesyltransferase inhibitors (FTIs) are small molecule signal transduction inhibitors that inhibit critical cell growth and survival pathways (reviewed by Karp and Lancet 2007). These agents are potent and selective competitive inhibitors of farnesyltransferase (FTase), an intracellular enzyme that catalyzes the transfer of the 15-carbon farnesyl moiety to a cysteine close to the C-terminus of a substrate polypeptide (End 1999; Basso et al 2006). A host of intracellular polypeptides including the small GTP-binding polypeptides of the Ras, Rho-B and Rheb families, membrane lamins, and centromeric proteins (CENPs) that interact with microtubules to promote the completion of mitosis, are substrates for prenylation via FTase (reviewed by Reuter et al 2000; Sebti and Der 2003; Karp and Lancet 2007). Inhibiting farnesylation of these polypeptides leads to diminished cell proliferation and, in some model systems, cell death. These cytotoxic effects have been attributed to FTI-induced inhibition of prosurvival signaling by Akt (Jiang et al 2000), the Rheb target mTOR (Castro et al 2003; Sebti and Adjei 2004; Witzig and Kaufmann 2006), or mitogen activated protein (MAP) kinases (reviewed by Reuter et al 2000; Morgan and Reuter 2006). Alternatively, it has been suggested that FTIs induce apoptosis by causing upregulation of the proapoptotic Bcl-2 family members Bax (Beaupre et al 2004), Bak (Feldkamp et al 1999), or PUMA (Gomez-Benito et al 2005).

While FTIs were initially developed on the premise that FT inhibition would prevent the post- translational processing of Ras proteins and thereby impede an early junctional process in overall signal transduction (reviewed by End 1999; Basso et al 2006), it is not surprising that their activity is not necessarily limited to ras mutated tumors nor is the effect equal among tumors bearing different mutated ras isoforms. Indeed, FTIs are by no means "selective" as they target proteins involved in disparate pathways and thereby exert their cytotoxic effects by interdicting multiple mechanisms of cellular survival, including angiogenesis, cellular adhesion, and inhibition of apoptosis. This notion is further substantiated by DNA microarray analyses of selected AML cell lines and primary AML marrow blasts, where tipifarnib modulates the expression of several gene networks, upregulating multiple genes involved in apoptosis, immunity, cell-cell adhesion and cytoskeletal organization, while downregulating genes involved in proliferation and cell cycle progression (Raponi et al 2004).
Hematologic malignancies provide a fertile testing ground for such agents because of the relative ease with which tumor tissue can be obtained throughout the therapeutic course. The ability to obtain target tissue in a longitudinal fashion provides a unique opportunity to define the relevant molecular components that may be modulated by these compounds and to relate those molecular effects to the clinical outcome. At present, three non-peptidomimetic FTIs are being tested clinically in a broad spectrum of hematologic malignancies: tipifarnib (R115777, Zarnestra), lonafarnib (SCH66336), and BMS-214662. To date, all three exhibit clinical and molecular biologic activities in diverse myeloid malignancies and MM with modest and acceptable toxicities. In particular, tipifarnib has exhibited clinical activity in patients with myeloid malignancies including elderly adults with AML who are not candidates for traditional cytotoxic chemotherapy (Karp et al 2001; Lancet et al 2007), patients with high-risk MDS (Kurzrock et al 2003, 2004; Fenaux et al 2007), myeloproliferative disorders (Mesa et al 2007), and imatinib-resistant chronic myelogenous leukemia (Cortes et al 2003). In this review, we will focus on the clinical development of tipifarnib for treatment of newly diagnosed AML, both as induction therapy for elderly adults with poor-risk AML and as maintenance therapy after achievement of first complete remission (CR) after induction and consolidation therapies for poor-risk AML.

\section{Clinical trials of tipifarnib as induction therapy Single-agent studies (Table I)}

The first clinical testing of FTIs in AML was a Phase I trial of the orally bioavailable FTI Tipifarnib administered for 21 days in patients with relapsed or refractory AML (Karp et al 2001). Consistent inhibition of FTase activity occurred at or above $300 \mathrm{mg}$ bid orally and dose-limiting toxicity (DLT), manifested as readily reversible central neurotoxicity, was observed at $1200 \mathrm{mg}$ bid. Oral absorption was rapid, with linear pharmacokinetics, and there was a dose-dependent increase in drug concentration in marrow with sustained levels 2- to 3-fold higher than concomitant levels in peripheral blood. Clinical responses were observed in 10 of 34 patients (29\%), including 2 complete remissions (CRs) in patients with relapsed AML, and occurred across all dosing levels (100-900 mg bid) without strict relationship to the degree of leukemic cell FTase inhibition. Intriguingly, responses were independent of ras mutational status, as none of the 34 leukemic samples demonstrated an N-ras mutation (Karp et al 2001). Expansion of these findings in 
Table I Trials of single agent tipifarnib in acute myelogenous leukemia

\begin{tabular}{|c|c|c|c|c|}
\hline Phase & Patient population & $\begin{array}{l}\text { Tipifarnib }^{\mathrm{a}} \\
\text { dose-schedule }\end{array}$ & Response rate & Author \\
\hline \multirow[t]{2}{*}{ I } & $\operatorname{Rel} / \operatorname{Ref}^{a}$ & $100-1200,21 / 28$ days & CR 8\% (2/34) & Karp (200I) \\
\hline & & MTD 900 & OR $29 \%(10 / 34)$ & \\
\hline \multirow[t]{2}{*}{1} & Rel/Ref & 400-1400, alternate week & CR 33\% (3/9) & Kirschbaum (2007) \\
\hline & & MTD I 200 & @ MTD & \\
\hline \multirow[t]{3}{*}{ II } & Rel/Ref & $600,21 / 28$ days & CR 7\% (11/169) & Harousseau (2007a) \\
\hline & & & sCR 4\% (6/I69) & \\
\hline & & & med OS I $2.2 \mathrm{mo}$ & \\
\hline \multirow[t]{4}{*}{ II } & New Dx & $600,21 / 28-63$ days & CR I4\% (22//58) & Lancet (2007) \\
\hline & Poor risk & & OR 23\% (37//58) & \\
\hline & Age $\geq 65$ & & med DFS $7.3 \mathrm{mo}$ & \\
\hline & & & CR med OS $18.6 \mathrm{mo}$ & \\
\hline \multirow[t]{3}{*}{ II } & New Dx & $300-600,21 / 28$ days & CR 6-15\% & Erba (2007) \\
\hline & Age $\geq 70$ & vs alternate week & CR I5\%/OR 20\% & \\
\hline & & & @ $300 \times 21 / 28$ days & \\
\hline \multirow[t]{3}{*}{ III } & New Dx & $600 \mathrm{mg}, 2 \mathrm{I} / 28$ days & CR 8\% (I8/228) & Harousseau (2007b) \\
\hline & Poor risk & vs BSC including HU & med DFS 8 mo & \\
\hline & Age $\geq 70$ & (vs 0\% BSC/HU arm) & CR med OS $20 \mathrm{mo}^{\mathrm{b}}$ & \\
\hline
\end{tabular}

${ }^{\mathrm{a}} \mathrm{mg}$ bid.

${ }^{b}$ no significant difference between tipifarnib and BSC arms with regard to OS.

Abbreviations: Rel/Ref, relapsed/refractory; CR, complete remission; sCR, sustained complete remission; OR, overall response; MTD, maximal tolerated dose; DFS, diseasefree survival; OS, overall survival; BSC, best supportive care; HU, hydroxyurea; mo, months.

an international Phase II study (Harousseau 2007a) yielded a CR rate of only $4 \%$ but, importantly, provided a template for subsequent studies of gene expression profiling to uncover determinants of response to tipifarnib (Raponi 2007).

Based on the Phase I findings (Karp 2001), Lancet et al (2007) conducted a unique Phase II study of tipifarnib administered at a dose of $600 \mathrm{mg}$ bid for 21 out of 28-63 days for 158 older adults with previously untreated, poor-risk AML. The median age was $74,75 \%$ had antecedent MDS, and $47 \%$ had adverse cytogenetics. Treatment-related mortality was $7 \%$. The CR rate in these elderly, poor-risk patients was $14 \%$ with an additional $10 \%$ partial response (partial remission, PR; hematologic improvement, HI). Among patients achieving $\mathrm{CR}, 82 \%$ had prior MDS and $40 \%$ had adverse cytogenetics. While median overall survival (OS) for all 158 patients was 5.3 months, median CR duration was 7.3 months and median OS for CR patients was 18.3 months. Patients who achieved PR or HI enjoyed a survival advantage as well, with a median OS 12.6 months. In contrast, median survival for patients who did not evince any type of response was 3.6 months. The lack of correlation between ras mutational status and clinical response was confirmed (Raponi et al 2008). Measurements of inhibition of farnesylation of the chaperone protein HDJ-2 in marrow blasts obtained on day 8 of tipifarnib therapy revealed an increase in unfarnesylated protein in $75 \%$ of marrow samples, while inhibition of farnesylation of the nuclear membrane protein Lamin A could be detected in $92 \%$ of all concomitantlyobtained samplers of buccal mucosa (Lancet et al 2007). This provocative finding may provide insight into potential mechanisms of FTI resistance, as discussed later in this review.

A randomized Phase III study of tipifarnib $600 \mathrm{mg}$ bid for 21 out of 28 days vs best supportive care (including hydroxyurea) of 457 adults over age 70 with newly diagnosed, poor risk AML who were not fit for conventional chemotherapy was recently completed in Europe and Canada (Harousseau et al 2007). Durable CR (median DFS 8 months, median OS 22 months) was achieved in $8 \%$ of those randomized to tipifarnib, while there were no CRs in the supportive care/hydroxyurea arm. Disappointingly, however, there was no statistically significant $O S$ benefit with tipifarnib treatment and the trial was deemed negative.

Explorations of alternative doses and schedules of tipifarnib are underway. The North American Intergroup conducted a Phase II 4-arm study of different doses (300 mg bid vs $600 \mathrm{mg}$ bid) and schedules (daily $\times 21$ days vs one week-on, one week-off) of single-agent tipifarnib as induction 
therapy for adults over age 70 with newly diagnosed AML (Erba et al 2007). While CRs and PRs were seen in all arms with acceptable toxicity profiles, the most salutary outcome was defined for those patients receiving tipifarnib $300 \mathrm{mg}$ bid for 21 days, with a $20 \%$ overall response rate $(15 \% \mathrm{CR}$, $5 \%$ PR) and a 1-year survival of roughly 30\%. Molecular analyses aimed at predicting response are planned. In a similar vein, a Phase I dose-escalation trial of a 1-week-on, 1-week-off schedule in adults with relapsed and refractory AML has defined the maximal tolerated dose (MTD) as $1200 \mathrm{mg}$ bid, with the DLT being renal toxicity, and achievement of CR in 3 of 9 treated at or near the MTD (Kirschbaum et al 2007).

\section{Combinatorial approaches (Table 2)}

While FTIs demonstrate reproducible activities as single agents, the resultant outcomes are modest. In order to improve the robustness of these results, the full development of FTIs in the therapeutic armamentarium for hematologic (and other) malignancies will require the design and testing of rational combinations of FTIs with cytotoxic, biologic and immunomodulatory agents in both the laboratory and the clinic. This is a burgeoning area of investigation in both the clinical and the laboratory arenas.

The combination of tipifarnib with conventional ara-C and anthracycline-based chemotherapy is under study by multiple investigators in newly diagnosed AML patients. In a Phase I-II study in 95 patients ages 15-70 (median age 50) with newly diagnosed AML or high-risk MDS, the MD Anderson group (Delmonte et al 2007) added tipifarnib $300 \mathrm{mg}$ bid to idarubicin plus ara-C (IA) induction and consolidation followed by 6 months of cyclical tipifarnib maintenance therapy. The addition of tipifarnib to IA induction yielded a $73 \% \mathrm{CR} / \mathrm{CRp}$ rate $(64 \% \mathrm{CR}, 9 \% \mathrm{CRp})$, which is identical to the group's data with IA alone in a historically similar population. With the addition of tipifarnib, CRs were achieved in $69 \%$ with adverse cytogenetics (13/19 of patients with $-5 /-7,21 / 35$ with other abnormalities) and 12/16 (75\%) with FLT-3 mutations. Major grade 3 toxicities included reversible diarrhea, rash and hyperbilirubinemia, induction mortality was very low. Median CR duration with tipifarnib is 17 months (12 month CR duration with IA alone) and the median OS is roughly the same as with similar historical controls (70 weeks vs 65 weeks, respectively). Additional observation and direct comparison between IA and IA plus tipifarnib in a prospective randomized Phase III trial will be needed to determine if the outcome with tipifarnib is superior to IA alone.

In a similar vein, a group led by investigators at Princess Margaret Hospital in Toronto, Ontario, Canada have conducted a Phase I dose-escalation study of tipifarnib added to conventional ara-C plus daunorubicin on days 6-15 of induction and consolidation therapies for newly diagnosed adults age 60 and older (Brandwein et al 2007), with achievement of CR in 9/22 (41\%) and $2 \mathrm{PR}$, for an overall response rate of $50 \%$. Of 7 with adverse cytogenetics 3 (43\%) achieved $\mathrm{CR}$ and 1 achieved PR. Tipifarnib dose escalation up to $600 \mathrm{mg}$ bid was well tolerated, albeit with mildly increased gastrointestinal toxicity, and a Phase II study with tipfiarnib at $600 \mathrm{mg}$ bid days 6-15 plus ara-C and daunorubicin is under development for adults $\geq 60$ years.

While it is possible that the antileukemic effects of tipifarnib might be enhanced by adjustment of the dose or

Table 2 Trials of tipifarnib in combination with chemotherapeutic agents

\begin{tabular}{|c|c|c|c|c|}
\hline \multirow[t]{2}{*}{ Phase } & \multirow{2}{*}{$\begin{array}{l}\text { Patient population } \\
\text { (Author) }\end{array}$} & \multicolumn{2}{|c|}{ Dose-schedules } & \multirow[t]{2}{*}{ Response rate } \\
\hline & & Tipifarnib $^{a}$ & Chemotherapy & \\
\hline \multirow[t]{4}{*}{ I } & New Dx & $300,400,600$ & etoposide & CR 25\% (2I/84) \\
\hline & Poor risk & |4 vs 2 | days & $100,150,200 \mathrm{mg}$ & med DFS 9.8 mo \\
\hline & Age $\geq 70$ & & Days $1-3$ and $8-10$ & CR med OS $22 \mathrm{mo}$ \\
\hline & (Karp 2006) & & & \\
\hline \multirow[t]{3}{*}{ I } & New Dx & $200-600$ & ara-C $100 \mathrm{mg} / \mathrm{m}^{2}$ day $\mathrm{Cl} \times 7$ & CR 4I\% (9/22) \\
\hline & Age $\geq 60$ & Days 6-15 & daunorubicin $60 \mathrm{mg} / \mathrm{m}^{2} /$ day $\times 3$ & OR $50 \%(11 / 22)$ \\
\hline & (Brandwein 2007) & & & \\
\hline \multirow[t]{3}{*}{ II } & New Dx & 300 & ara-C $1.5 \mathrm{~g} / \mathrm{m}^{2} /$ day $\mathrm{Cl} \times 3-4$ & CR 69\% (70/95) \\
\hline & Age |7-6I & & idarubicin $12 \mathrm{mg} / \mathrm{m}^{2} /$ day & med DFS I7 mo \\
\hline & (Delmonte 2007) & & & \\
\hline
\end{tabular}

${ }^{\mathrm{a}} \mathrm{mg}$ bid.

Abbreviations: $C R$, complete remission; $C R p$, complete remission with incomplete platelet recovery; DFS, disease-free survival; OR, overall response; OS, overall survival; mo, months. 
schedule, an alternative approach would be to combine this agent with existing antileukemic therapy. Toward this end, preclinical studies have revealed that the antiproliferative effects in human AML cells in vitro are additive when tipifarnib is combined with cladrabine or fludarabine (Korycka et al 2004) and synergistic when tipifarnib is combined with bortezomib (Yanamandra et al 2006) or daunorubicin (Medeiros et al 2007). Interestingly, tipifarnib appears to inhibit the drug efflux activity of the prototypical drug resistance protein P-glycoprotein (P-gp) in a dose-dependent fashion in human acute T-lymphoblastic leukemia and AML cell lines in a manner that is independent of its FT inhibitory activity (Medeiros 2007). This P-gp inhibition may be a part of the synergistic interaction between tipifarnib and daunorubicin and, at least in theory, could apply to combinations of tipifarnib with other P-gp substrates such as etoposide (Mahadevan and List 2004; Medeiros et al 2007). Moreover, the ability of tipifarnib to modulate P-gp activity and the possibility that such modulation could contribute to synergy with antileukemic agents such as daunorubicin and etoposide may have special relevance to elderly patients with AML, where high levels of P-gp expression are common and correlate with clinical drug resistance (Leith et al 1999; Appelbaum et al 2006).

The topoisomerase II poison etoposide, which stabilizes covalent adducts between the nuclear enzyme topoisomerase II and DNA (Li and Liu 2001; Wang 2002), has been used alone and in combination for acute leukemias and has demonstrated efficacy, particularly when combined with other cytotoxic agents (Evans and Prentice 1982; Bishop et al 1990; Bolanos-Meade et al 2003). Etoposide exhibits limited activity as a single agent when given intravenously in doses of $200-400 \mathrm{mg} / \mathrm{m}^{2}$ daily $\times 5$ and orally at daily doses of 200-500 $\mathrm{mg} \times 5-7$ days (Evans and Prentice 1982). Oral etoposide at doses of $75-125 \mathrm{mg} / \mathrm{m}^{2}(100-200 \mathrm{mg})$ daily $\times$ 3-5 has been combined with oral idarubicin at doses of $20-30 \mathrm{mg} / \mathrm{m}^{2}$ daily $\times 3-5$ as induction therapy for small numbers of selected AML patients $>55$ years (Jackson et al 1997; Oriol et al 2003; Fiegl et al 2005), with CR rates ranging from $0 \%$ to $30 \%$ but with short CR durations (Jackson et al 1997; Fiegl et al 2005) and significant treatment-related toxicity and death (Oriol et al 2003; Fiegl et al 2005).

A multicenter Phase I trial of oral tipifarnib plus oral etoposide $(T+E)$ was designed with escalating doses of both drugs and exploration of two different durations of tipifarnib administration (14 vs 21 days) to examine the safety and tolerability of $\mathrm{T}+\mathrm{E}$ in patients $\geq 70$ years who were not candidates for conventional induction chemotherapy and to preliminarily assess the antileukemic activity of the combination (Karp et al 2006). A total of 84 patients (median age 77 ) received 224 cycles of tipifarnib (300-600 mg bid for 14 or 21 days) + etoposide (100-200 mg daily on days 1-3 and 8-10). All patients had at least 1 poor-risk feature in addition to age, including secondary AML (55\%), adverse cytogenetics (54\%), and/or 3 or more non-hematologic co-morbidities (45\%). In the previous phase II study of single-agent tipifarnib administered at $600 \mathrm{mg}$ bid for 21 consecutive days, grade 3 or 4 adverse events occurred in $47 \%$ of in elderly AML patients (Lancet et al 2007). When the same 21-day schedule of tipifarnib was combined with etoposide, grade 4 oral mucositis, grade 3 hyperbilirubinemia, and multi-organ failure were detected at tipifarnib doses of $400 \mathrm{mg}$ twice daily, independent of etoposide dose. In contrast, the same etoposide schedule was much better tolerated when tipifarnib was administered for 14 days without any dose limiting toxicities, even at a tipifarnib dose of $600 \mathrm{mg}$ bid. Oral mucositis, a well-known toxicity of etoposide, has not accompanied previous studies of single-agent tipifarnib (Karp et al 2001; Kurzrock et al 2004; Fenaux et al 2007; Lancet et al 2007) The apparent relationship between tipifarnib schedule and mucositis induction may represent synergy between tipifarnib and etoposide against normal oral mucosa. On the other hand, tipifarnib-related neurotoxicity was not enhanced by combination with etoposide.

For the entire group of 84 patients, the CR rate was $25 \%$, with median CR duration being 9.8 months (range 3-31 months) and median OS being 22 months (range 3.5-36+), with $67 \%$ surviving for more than 1 year and $43 \%$ alive at $16+$ to $36+$ months. When the relationship between dose or schedule and response was examined, CR was achieved in $31 \%$ of the patients receiving tipifarnib doses of at least $400 \mathrm{mg}$ twice daily and in 16/54 (30\%) patients receiving 14-day $\mathrm{T}$ vs 5/30 (17\%) receiving 21-day. Interestingly, CR rates of $50 \%$ (3/6 patients per cohort) were achieved in three of the 14-day cohorts where tipifarnib dose was at least $400 \mathrm{mg}$ twice daily in combination with etoposide at 100 or $200 \mathrm{mg}$ daily. Thus, this combination has yielded encouraging clinical results, with a CR rate that is almost double that achieved with tipifarnib alone (Lancet et al 2007) in a group of patients for whom traditional antileukemia chemotherapy may not be appropriate because of advanced age, poor risk biologic disease features, and/or the presence of significant non-hematologic co-morbidities (Appelbaum et al 2006; Estey 2007). In view of the results observed, a randomized multicenter Phase II trial is planned 
to compare two 14-day cohorts to select the optimal doses of $\mathrm{T}+\mathrm{E}$ that will produce the maximal $\mathrm{CR}$ rate with the most acceptable toxicity profile.

\section{Treatment of minimal residual disease: maintenance therapy}

Maintenance chemotherapy in CR after multiple cytotoxic regimens is effective in the setting of acute lymphoblastic leukemia but has not yet proven to lengthen CR in AML, particularly in older patients or those with poor-risk features (Cassileth et al 1992; Lowenberg et al 1998; Goldstone et al 2001; Buchner et al 2006; Estey 2007). Nonetheless, the concept of maintenance therapy after intensive therapy is appealing if indeed such therapy could prolong CR or prevent relapse without incurring serious toxicities or clonal evolution of remaining leukemic cells. It is becoming increasingly possible to identify AML patients whose CR durations are predicted to be short, based on clinical and biologic features that reflect inherent drug resistance (Harris et al 1999; Grimwade et al 2001; Arber et al 2003; Bullinger et al 2004; Yanada et al 2005; Radmacher et al 2006; Estey et al 2007; Mrozek et al 2007). Indeed, in adults with one or more poor-risk features, the median CR duration is $\leq 9$ months and the 1-year DFS is 20\% (Archimbaud et al 1999; Goldstone et al 2001; Stone et al 2001; Bolanos-Meade et al 2003; Breems et al 2005; Estey 2007). In such patients, the so-called "minimal residual disease" (MRD) state should provide a fertile testing ground for new approaches aimed at prolonging $\mathrm{CR}$ and preventing or deterring relapse.

In this regard, it is reasonable to speculate that FTIs might suppress regrowth of the malignant clone when the residual tumor burden after cytotoxic chemotherapies has been reduced to a minimal state. This concept has now been tested in patients with AML with poor-risk features in first CR who are expected to relapse in less than 1 year (age $\geq 60$ and/or secondary AML or adverse cytogenetics) (Karp et al 2008). In a Phase II trial of tipifarnib monotherapy for 48 such adults with poor-risk AML in first CR, tipifarnib $400 \mathrm{mg}$ bid for 14 out of 21 days was initiated after recovery from consolidation chemotherapy, for a maximum 16 cycles. Twenty (42\%) completed 16 cycles, 24 (50\%) were removed from study for relapse, and 4 (8\%) discontinued drug prematurely for intolerance. Non-hematologic toxicities were rare, but tipifarnib dose was reduced in 58\% for myelosuppression. Median DFS was 13.5 months (range 3.5-60+), with $30 \%$ having DFS $\geq 2$ years. When CR durations for patients who received tipifarnib maintenance were compared with the CR durations of historically similar patients who received the same induction and consolidation therapies but did not receive tipifarnib, DFS for patients with secondary AML $(p=0.01)$, adverse cytogenetics $(p=0.07)$, or both poor-risk features $(p=0.02)$ appeared to be prolonged with tipifarnib. This effect of tipifarnib for patients with secondary AML and/or adverse cytogenetics is consistent with previous demonstrations of tipifarnib activity in patients with highrisk MDS (Fenaux et al 2007) and in elderly patients with MDS/AML including those with adverse cytogenetics (Karp et al 2006; Lancet et al 2007). On the other hand, historical comparison suggested that tipifarnib maintenance may not prolong DFS in patients whose sole risk factor was age $\geq 60$ and whose AML did not exhibit poor risk biology. A randomized trial, as is currently underway in the Eastern Cooperative Oncology Group (ECOG), will be needed to demonstrate any definitive benefit of tipifarnib as post-remission maintenance therapy for any subgroups of patients with AML with poorrisk features, in particular those with secondary AML and/or adverse cytogenetics.

One future goal is to determine who will benefit from the addition of tipifarnib in the MRD setting. This may be particularly challenging in patients with normal cytogenetics, where clinical outcomes and cure rates can vary dramatically, reflecting heterogeneity on the molecular level (Bullinger et al 2004; Wilson et al 2006; Mrozek et al 2007). In this regard, recent studies have defined single gene mutations and gene expression signatures that may help to discriminate prognostic subgroups in cytogenetically "normal" AML (Radmacher et al 2006; Mrozek et al 2007). In sum, the results of this limited Phase II study suggest that some patients with poor-risk AML, including those with secondary AML and/or adverse cytogenetics, may benefit from tipifarnib maintenance therapy without incurring clinically or biologically significant risks. Future studies of this approach should examine alternative tipifarnib dosing (eg, reduced dosing, different schedules) and continuation of therapy beyond 16 cycles, as well as a randomized, placebo-controlled trial of tipifarnib maintenance therapy. Finally, stratification based on molecular features may refine the ability to target the subset of patients who stand to derive the greatest benefit from the tipifarnib maintenance approach.

\section{Resistance to tipifarnib}

Malignant cells have an uncanny ability to become resistant to new antitumor agents, and it is likely that the FTIs will be no exceptions. Such resistance, whether intrinsic or acquired, limits net drug efficacy. To date, resistance to FTIs has been demonstrated in diverse cell lines that have been 
developed via selective adaptation (Prendergast et al 1996; Smith et al 2002; Bruzek et al 2005; Buzzeo et al 2005; Zhang et al 2005) or have been genetically engineered to express FT enzyme mutations (Del Villar et al 1999; Raz et al 2007). For example, a tipifarnib-resistant human colon cancer cell line, generated by continuous drug exposure, exhibits a marked reduction in the FT enzyme itself without enzyme mutations or aberrations in activation of enzyme subunits (Prendergast et al 1996). In a similar construct, a lonafarnibresistant $\mathrm{Ph}+\mathrm{ALL}$ cell line from transgenic mice has been generated by growing those cells on stroma in the presence of increasing lonafarnib concentrations (Zhang et al 2005). The resultant lonafarnib-resistant cells exhibit marked increase in gene expression of the novel ATP binding cassette (ABC) transporter homolog ATP11a and are, interestingly, relatively resistant to imatinib as well. A novel MM line derived from 8226 (8226/R5) exhibits resistance to both tipifarnib and bortezomib, with such resistance unrelated to FTase mutations, drug transporters, or heat shock protein expression (Buzzeo et al 2005). In comparison with drug sensitive 8226 cells, gene expression profiling of the resistant 8226/R5 cells revealed increased expression of Jak2 and Stat 1 with marked elevation in both phosphorylated Stat1 and Stat3 and, as a result of activated Stat3, an increase in BCL-X $\mathrm{L}_{\mathrm{L}} \mathrm{mRN}$ and protein expression. In addition, 8226/R5 cells were found to overexpress a particular isoform of the $\mathrm{PI}_{3} \mathrm{~K}$ p110 subunit. It is reasonable to speculate that overexpression of key components of the $\mathrm{PI}_{3} / \mathrm{AKT}$ anti-apoptosis pathway by any mechanism might be expected to confer relative resistance to FTI-induced apoptosis. Indeed, this appears to be the case in the generation of resistance to lonafarnib in the HCT116 human colon cancer cell line, which appears to relate to an increase in Akt and/or its downstream intermediaries (Bruzek et al 2005). Whether or not any of these mechanisms of resistance are clinically relevant is not yet known. The elucidation of these and other mechanisms of resistance at both cellular and humoral levels of drug disposition is an important aspect of the ongoing clinical development of FTIs.

In the clinical arena, Goemans et al (2005) examined drug sensitivity profiles of pediatric leukemias, using a methylthiazole-tetrazolium (MTT) assay to compare tipifarnib responsiveness with traditional cytotoxic agents. T-cell ALLs and acute monoblastic AMLs exhibited the greatest tipifarnib sensitivity without correlation between ras mutational status or in vitro drug responsiveness but with correlation in AML samples between resistance to tipifarnib and resistance to anthracyclines or etoposide. These studies may be a useful template for understanding shared mechanisms of drug resistance among structurally diverse compounds and, in turn, providing insights into strategies by which to overcome such resistance factors.

\section{Gene expression: insights into FTI mechanisms of action and prediction of response}

The ability to identify molecular predictors of response to specific drugs or drug combinations should direct the selection of a therapeutic approach that is most likely to induce meaningful clinical response. Toward this end, microarray technology provides a spectrum of gene expression signatures in AML which, in turn, affords a molecular stratification of patients with respect to disease biology and clinical outcome. Raponi et al (2004) have conducted serial studies of gene expression profiling in the context of AML cell lines and primary AML marrow cells exposed to tipifarnib in vitro, in marrow blasts from patients with relapsed and refractory AML undergoing treatment with tipifarnib alone (Harousseau et al 2007a; Raponi et al 2007), and most recently in marrow blasts from elderly adults with previously untreated AML with poor-risk features who received tipifarnib alone as induction therapy (Lancet et al 2007; Raponi et al 2008). Each of these studies has identified networks of differentially expressed genes and gene combinations capable of predicting response to single agent tipifarnib. The in vitro studies identified integrated gene networks whose activities are modulated in an orchestrated fashion to yield net cell death in diverse AML cell lines and in primary AML marrow samples, while the studies in AML cells from patients with relapsed and refractory AML identified a signature of 8 genes that are differentially expressed in responders vs nonresponders, with overexpression of one gene in particular, the lymphoid blast crisis oncogene (oncoLBC or AKAP13), capable of accurately predicting clinical response to tipifarnib (Raponi et al 2007). Provocatively, the AKAP13 protein acts as a guanine nucleotide exchange factor for Rho proteins (Zheng et al 1995; Toksoz et al 2004) and contains a region that is homologous to an $\alpha$-helical domain known to interact with nuclear envelope protein lamin B (Foisner et al 1991). This is especially intriguing because Rho and lamin proteins are farnesylated, and AKAP13 activates both types of proteins. The recent discovery that lamin B is critical to the assembly of the mitotic spindle (Tsai et al 2006) makes it tempting to speculate that AKAP13 might have an indirect role in promoting or permitting completion of mitosis, perhaps in concert with another group of FTI targets, namely CENPs. Finally, gene expression profiles of AML marrow blasts 
from elderly adults with newly diagnosed, poor-risk AML (Raponi et al 2008) demonstrate that response to tipifarnib relates to the expression of two specific genes: up-regulation of the gene encoding the guanine nucleotide exchange factor RASGRP1, which activates Ras, and down-regulation of $A P T X$, the gene encoding the DNA excision repair protein aprataxin (Ahel et al 2006). Conversely, active aprataxin may be able to repair damaged DNA in a way that uncouples the detection of that damage and the eventual completion of programmed cell death. It should be noted that the studies to date have been conducted in relatively small numbers of patients and may therefore be somewhat biased. Nonetheless, the continuing efforts to compare gene expression profiles of AML marrow blasts obtained pretreatment and during treatment with tipifarnib alone or in combination with other agents hold promise for identifying genomic markers that could accurately predict susceptibility vs resistance to tipifarnib-based therapies.

\section{Perspectives for continuing clinical development of tipifarnib}

In summary, tipifarnib inhibits malignant cell growth and survival by interfering with multiple intracellular signaling pathways. As a single agent, it has reproducible clinical effects on AML and other hematologic malignancies, but to date those effects are detected in a minority of patient subgroups. There are a number of potential explanations for the less-than-robust clinical results with FTIs as single agents, including inherent patient and disease heterogeneity, potential recruitment of survival pathways including DNA repair, and activation of other post-translational processing enzymes (eg, geranylgeranyltransferase) that might circumvent the effects of FT inhibition. As with all other malignancies, the optimal approach is likely to lie in rational combinations of tipifarnib or other FTIs with cytotoxic, biologic, and/or immunomodulatory agents with non-cross-resistant mechanisms of action. The clinical trials currently in progress will provide the critical foundations for defining the optimal roles of tipifarnib in patients with diverse hematologic malignancies.

The precise mechanisms by which tipifarnib exerts its cytotoxicity remain to be defined. The original notion that these agents targeted Ras mutations is clearly not complete, and it is likely that all FTIs have an impact on multiple molecules and pathways involved in cellular integrity. Studies to define the mechanisms by which tipifarnib alters cellular metabolism and modulate the activities of specific signaling pathways in normal and malignant precursors are a pivotal part of this effort. In this regard, the potential impact of FTIs on other disease processes is presaged by the ability of ABT-100 to ameliorate the process of premature aging in a mouse model of progeria which, like the human condition, is characterized by accumulation of an abnormally farnesylated form of the lamin A precursor prelamin A, disruption of orderly nuclear scaffolding, and resultant misshapen nuclei (Fong et al 2006). The clinical and correlative laboratory trials in progress and under development will provide the critical foundations for defining the optimal roles of tipifarnib and other FTIs in patients with cancer, and perhaps other diseases of disordered cellular metabolism as well.

\section{Disclosures}

Neither author has any conflicts of interest to disclose.

\section{References}

Ahel I, Rass U, El-Khamisy SF, et al. 2006 The neurodegenerative disease protein aprataxin resolves abortive DNA ligation intermediates. Nature, 443:713-6.

Appelbaum FR, Gundacker H, Head DR, et al. 2006 Age and acute myeloid leukemia. Blood, 107:3481-5.

Arber DA, Stein AS, Carter NH, et al. 2003. Prognostic impact of acute myeloid leukemia classification. Importance of detection of recurring cytogenetic abnormalities and multilineage dysplasia on survival. Am J Clin Pathol, 119:672-80.

Archimbaud E, Jehn U, Thomas X, et al. 1999. Multicenter randomized phase II trial of idarubicin vs mitoxantrone, combined with VP16 and cytarabine for induction/consolidation therapy, followed by a feasibility study of autologous peripheral blood stem cell transplantation in elderly patients with acute myeloid leukemia. Leukemia, 13:843-9.

Basso AD, Kirschmeier P, Bishop WR. 2006. Farnesyltransferase inhibitors. $J$ Lipid Res, 47:15-31.

Beaupre DM, Cepero E, Obeng EA,et al. 2004. R115777 induces Ras-independent apoptosis of myeloma cells via multiple intrinsic pathways. Mol Cancer Therap, 3:179-86.

Bishop JF, Lowenthal RM, Joshua D, et al. 1990. Etoposide in acute nonlymphocytic leukemia. Australian Leukemia Study Group. Blood, 75:27-32.

Bolanos-Meade J, Karp JE, Guo C, et al. 2003. Timed sequential therapy of acute myelogenous leukemia in adults: a phase II study of retinoids in combination with the sequential administration of cytosine arabinoside, idarubicin and etoposide. Leuk Res, 27:313-21.

Brandwein JM, Leber BF, Howson-Jan K, et al. 2007. A Phase I study of tipifarnib in combination with conventional induction and consolidation therapy for previously untreated patients with acute myeloid leukemia age 60 and over [abstract]. Blood, 110:274a (abstract 899).

Breems DA, Van Putten WLJ, Huijgens PC, et al. 2005. Prognostic index for adult patients with acute myeloid leukemia in first relapse. J Clin Oncol, 23:1969-78.

Bruzek LM, Poynter JN, Kaufmann SH, et al. 2005. Characterization of a human carcinoma cell line selected for resistance to farnesyltransferase inhibitors. Mol Pharmacol, 68:477-86.

Buchner T, Berdel WE, Schoch C, et al. 2006. Double induction containing either two courses or one course of high-dose cytarabine plus mitoxantrone and postremission therapy by either autologous stem-cell transplantation or by prolonged maintenance for acute myeloid leukemia. J Clin Oncol, 24:2480-9. 
Bullinger L, Dohner K, Bair E, et al. 2004. Use of gene-expression profiling to identify prognostic subclasses in adult acute myeloid leukemia. New Engl J Med, 350:1605-16.

Buzzeo R, Enkemann S, Nimmanapalli R, et al. 2005. Characterization of a R115777-resistant human multiple myeloma cell line with crossresistance to PS-341. Clin Cancer Res, 11:6057-64

Cassileth PA, Lynch E, Hines JD, et al. 1992. Varying intensity of postremission therapy in acute myeloid leukemia. Blood, 79:1924-30.

Castro AF, Rebhun JF, Clark GJ, et al. 2003 Rheb binds tuberous sclerosis complex 2 (TSC2) and promotes S6 kinase activation in a rapamycinand farnesylation-dependent manner. J Biol Chem, 278:32493-6.

Cortes J, Albitar M, Thomas D, et al. 2003. Efficacy of the farnesyl transferase inhibitor R115777 in chronic myeloid leukemia and other hematologic malignancies. Blood, 101:1692-7.

Delmonte J, Kantarjian HM, Garcia-Manero G, et al. 2007. Final update of Phase I-II study of the farnesyltransferase inhibitor tipifarnib in combination with idarubicin and cytarabine for patients with newly diagnosed acute myeloid leukemia or high-risk myelodysplasia [abstract]. Blood, 110:136a (abstract 441).

Del Villar K, Urano J, Guo L, et al. 1999. A mutant form of protein farnesyltransferase exhibits increase resistance to farnesyltransferase inhibitors. J Biol Chem, 274:27010-17.

End DW. 1999. Farnesyl protein transferase inhibitors and other therapies targeting the Ras signal transduction pathway. Invest New Drugs, 17:241-58

Erba HP, Kopecky KJ, Kirschbaum MH, et al. 2007. Phase II studies of different schedules and doses of the farnesyltransferase inhibitor tipifarnib (R115777, Zarnestra, NSC 702818) for patients of age 70 or older with previously untreated acute myeloid leukemia (AML): A North American Intergroup study (S0432) [abstract]. Blood, 110:136a (abstract 440).

Estey E. 2007. Acute myeloid leukemia and myelodysplastic syndromes in older patients. $J$ Clin Oncol, 25:1908-15.

Evans JPM, Prentice HG. 1982. Remission induction in acute myeloid leukaemia with oral etoposide. Lancet, 2(8311):1345-6.

Feldkamp MM, Lau N, Guha A. 1999. Growth inhibition of astrocytoma cells by farnesyl transferase inhibitors is mediated by a combination of anti-proliferative, pro-apoptotic and anti-angiogenic effects. Oncogene, 18:7514-26.

Fenaux P, Raza A, Mufti G, et al. 2007. A multicenter phase 2 study of the Farnesyltransferase inhibitor tipifarnib in intermediate- to high-risk myelodysplastic syndrome. Blood, 109:4158-63.

Fiegl M. Buchner T, Hiddemann W, et al. 2005. A phase I/II study of oral etoposide and idarubicin in elderly patients with high-risk acute myeloid leukemia unable to undergo intensive chemotherapy. Ann Hematol, 84:227-31.

Foisner R, Traub P, Wiche G. 1991. Protein kinase A- and protein kinase C-regulated interaction of plectin with lamin $\mathrm{B}$ and vimentin. Proc Natl Acad Sci USA, 88:3812-16.

Fong LG, Frost D, Meta M, et al. 2006. A protein Farnesyltransferase inhibitor ameliorates disease in a mouse model of progeria. Science, 311:1621-3.

Goemans BF, Zwaan CM, Harlow A, et al. 2005 In vitro profiling of the sensitivity of pediatric leukemia cells to tipifarnib: identification of T-cell ALL and FAB M5 AML as the most sensitive subsets. Blood, 106:3532-7.

Goldstone AH, Burnett AK, Wheatley K, et al. 2001. Attempts to improve treatment outcomes in acute myeloid leukemia (AML) in older patients: the results of the United Kingdom Medical Research Council AML 11 trial. Blood, 98:1302-11.

Gomez-Benito M, Marzo I, Anel A, et al. 2005. Farnesyltransferase inhibitor BMS-214662 induces apoptosis in myeloma cells through PUMA up-regulation, Bax and Bak activation, and Mcl-1 elimination. Mol Pharmacol, 67:1991-8.

Grimwade D, Walker H, Harrison G, et al. 2001. The predictive value of hierarchical cytogenetic classification in older adults with acute myeloid leukemia (AML): analysis of 1065 patients entered into the United Kingdom Medical Research Council AML 11 trial. Blood, 98:1312-20
Harris NL, Jaffe ES, Diebold J, et al. 1997. World Health Organization classification of neoplastic diseases of the hematopoeitic and lymphoid tissues: report of the clinical advisory committee meeting - Airlie House, Virginia, November. J Clin Oncol, 17:3835-49.

Harousseau J-L, Lancet JE, Reiffers J, et al. 2007a. A phase 2 study of the oral farnesyltransferase inhibitor tipifarnib in patients with refractory or relasped acute myeloid leukemia. Blood, 109:5151-6.

Harousseau J-L, Martinelli G, Jedrejczak WW, et al. 2007b. A randomized Phase 3 study of tipifarnib compared to best supportive care (including hydroxyurea) in the treatment of newly diagnosed acute myeloid leukemia (AML) in patients 70 or older. Blood, 110:135a (abstract 439).

Jackson GH, Taylor PR, Iqbal A, et al. 1997. The use of an all oral chemotherapy (idarubicin and etoposide) in the treatment of acute myeloid leukaemia in the elderly: a report of toxicity and efficacy. Leukemia, 11:1193-6.

Jiang K, Coppola D, Crespo NC, et al. 2000. The phosphoinositide 3-OH kinase/AKT2 pathway as a critical target for farnesyltransferase inhibitor-induced apoptosis. Mol Cell Biol, 20:139-48.

Karp JE, Lancet JE, Kaufmann SH, et al. 2001. Clinical and biologic activity of the farnesyltransferase inhibitor R115777 in adults with refractory and relapsed acute leukemias: a phase I clinical-laboratory correlative trial. Blood, 97:3361-9.

Karp JE, Feldman EJ, Morris LE, et al. 2006. Active oral regimen for elderly adults with newly diagnosed acute myelogenous leukemia (AML): phase I trial of oral tipifarnib (T) combined with oral etoposide (E) for adults $\geq$ age 70 who are not candidates for traditional cytotoxic chemotherapy. Blood, 108:130a.

Karp JE, Lancet JE. 2007. Development of farnesyltransferase inhibitors for clinical cancer therapy: focus on hematologic malignancies. Cancer Investig, 25:484-94.

Kirschbaum MH, Selwyn Stein A, Tuscano J, et al. 2007. A Phase I study of the farnesyltransferase inhibitor tipifarnib in a week-on week-off dose schedule in acute myelogenous leukemia [abstract]. Blood, 110:182a (abstract 891).

Korycka A, Smolewski P, Robak T. 2004. The influence of farnesyl protein transferase inhibitor R115777 (Zarnestra) alone and in combination with purine nucleoside analogs on acute myeloid leukemia progenitors in vitro [abstract]. Eur J Haematol, 73:418-26.

Kurzrock R, Kantarjian HM, Cortes JE, et al. 2003. Farnesyltransferase inhibitor R115777 in myelodysplastic syndrome: clinical and biologic activities in the Phase 1 setting. Blood, 102:4527-34.

Kurzrock R, Albitar M, Cortes JE, et al. 2004. Phase II study of R115777, a farnesyltransferase inhibitor, in myelodysplastic syndrome. $J$ Clin Oncol, 22:1287-92.

Lancet JE, Willman CL, Bennett JM. 2000. Acute myelogenous leukemia and aging: clinical interactions. Hematol Oncol North Am, 14:251-67.

Lancet JE, Gojo I, Gotlib J, et al. 2007. A phase II study of the Farnesyltransferase inhibitor Tipifarnib in elderly patients with previously untreated poor-risk acute myelogenous leukemia. Blood, 109:1387-94.

Leith CP, Kopecky KJ, Chen IM, et al. 1999. Frequency and clinical significance of the expression of the multidrug resistance proteins MDR1/P-glycoprotein, MRP1, and LRP in acute myeloid leukemia: a Southwest Oncology Group study. Blood, 94:1086-99.

Li TK, Liu LF. 2001. Tumor cell death induced by topoisomerase-targeting drugs. Annu Rev Pharmacol Toxicol, 41:53-77.

Lowenberg B, Suici S, Archimbaud E, et al. 1998. Mitoxantrone versus daunorubicin in induction-consolidation chemotherapy - the value of low-dose cyatarbine for maintenance of remission, and an assessment of prognostic factors in acute myeloid leukemia in the elderly: final report of the Leukemia Cooperative Group of the European Organization for the Research and Treatment of Cancer and the Dutch-Belgian Hemato-Oncology Cooperative Hovon Group randomized phase III study AML-9. J Clin Oncol, 16:872-81.

Mahadevan D, List AF. 2004. Targeting the multidrug resistance-1 transporter in AML: molecular regulation and therapeutic strategies. Blood, 104:1940-51. 
Manning BD, Cantley LC. 2003. Rheb fills a GAP between TSC and TOR. Trends Biochem Sci, 28:573-6.

Mrozek K, Marcucci G, Paschka P, et al. 2007. Clinical relevance of mutations and gene-expression changes in adult acute myeloid leukemia with normal cytogenetics: are we ready for a prognostically prioritized molecular classification? Blood, 109:431-48.

Medeiros BC, Landau HJ, Morrow M, et al. 2007. The farnesyl transferase inhibitor, tipifarnib, is a potent inhibitor of the MDR1 gene product, P-glycoprotein, and demonstrates significant cytotoxic synergism against human leukemia cell lines. Leukemia, 21:739-46.

Mesa RA, Camoriano JK, Geyer SM, et al. 2007. A Phase II trial of tipifarnib in myelofibrosis: primary, post-polycythemia vera and post-essential thrombocythemia. Leukemia, 21:1964-70.

Morgan MA, Reuter CW. 2006. Molecularly targeted therapies in myelodysplastic syndromes and acute myeloid leukemias. Ann Hematol, 85:139-63.

Oriol A, Ribera J-M, Brunet S, et al. 2003. Oral induction and consolidation chemotherapy with idarubicin and etoposide in elderly patients with acute myeloid leukemia. Haematologica, 88:229-30.

Prendergast GC, Davide JP, Lebowitz PF, et al. 1996.. Resistance of a variant ras-transformed cell line to phenotypic reversion by farnesyltransferase inhibitors. Cancer Res, 56:2626-32.

Radmacher MD, Marcucci G, Ruppert AS, et al. 2006. Independent confirmation of a prognostic gene-expression signature in adult acute myeloid leukemia with a normal karyotype: a Cancer and Leukemia Croup B study. Blood, 108:1677-83.

Raponi M, Belly RT, Karp JE, et al. 2004. Microarray analysis reveals genetic pathways modulated by tipifarnib in acute myeloid leukemia. BMC Cancer, 4:56.

Raponi M, Harousseau JL, Lancet JE, et al. 2007. Identification of molecular predictors of repsonse in a study of tipifarnib treatment in relapsed and refractory acute myelogenous leukemia. Clin Cancer Res, 13:2254-60.

Raponi M, Lancet JE, Fan H, et al. 2008. A two-gene classifier for predicting response to the farnesyltransferase inhibitor tipifarnib in acute myeloid leukemia. Blood, 111:2589-96.

Raz T, Nardi V, Azam M, et al. 2007. Resistance to the farnesyl transferase inhibitor SCH66336 (Lonafarnib) caused by mutations in the target protein farnesyl transferase beta. Blood, 110:2102-9.

Reuter CWM, Morgan MA, Bergmann L. 2000. Targeting the Ras signaling pathway: a rational, mechanism-based treatment for hematologic malignancies? Blood, 96:1655-69.
Sebti SM, Der CJ. 2003. Searching for the elusive targets of Farnesyltransferase inhibitors. Nat Rev, 3:945-51.

Sebti SM, Adjei AA. 2004. Farnesyltransferase inhibitors. Semin Oncol, 31:28-39.

Smith V, Rowlands MG, Barrie E, et al. 2002. Establishment and characterization of acquired resistance to farnesyl protein transferase inhibitor R115777 in a human colon cancer cell line. Clin Cancer Res, 8:2002-9.

Stone RM, Berg DT, George SL, et al. 2001. Postremission therapy in older patients with de novo acute myeloid leukemia: a randomized trial comparing mitoxantrone and intermediate-dose cytarabine with standard-dose cytarabine. Blood, 98:548-53.

Toksoz D, Williams DA. 1994. Novel human oncogene lbc detected by transfection with distinct homology regions to signal transduction proteins. Oncogene, 9:621-8.

Tsai MY, Wang S, Heidinger JM, et al. 2006. A mitotic lamin B matrix induced by RanGTP required for spindle assembly. Science, 311:1887-93.

Wang JC. 2002. Cellular roles of DNA topoisomerases: a molecular. Nat Rev Mol Cell Biol, 3:430-40.

Wilson CS, Davidson GS, Martin SB, et al. 2006. Gene expression profiling of adult acute myeloid leukemia identifies novel biologic clusters for risk classification and outcome prediction. Blood, 108:685-96.

Witzig TE, Kaufmann SH. 2006. Inhibition of the phosphatidylinositol3-kinase/mammalian target of rapamycin pathway in hematologic malignancies. Curr Treat Options Oncol, 7:285-94.

Yanada M, Suzuki M, Kawashima K, et al. 2005. Long-term outcomes for unselected patients with acute myeloid leukemia categorized according to the World Health Organization classification: a single center experience. Eur J Hematol,74:418-23.

Yanamandra N, Colaco NM, Parquet NA, et al. 2006. Tipifarnib and bortezomib are synergistic and overcome cell adhesion-mediated drug resistance in multiple myeloma and acute myeloid leukemia. Clin Cancer Res, 12:591-9.

Zhang B, Groffen J, Heisterkamp N. 2005. Resistance to farnesyltransferase inhibitors in Bcr/Abl-positive lymphoblastic leukemia by increased expression of a novel ABC transporter homolog ATP11a. Blood, 106:1355-61.

Zheng Y, Olson MF, Hall A, et al. 1995. Direct involvement of the small GTP-binding protein Rho in lbc oncogene function. J Biol Chem, 270: 9031-4. 